\title{
Robust Step Detection from Different Waist-Worn Sensor Positions: Implications for Clinical Studies
}

\author{
Matthias Tietsch ${ }^{a, b} \quad$ Amir Muaremi $^{a}$ leuan Clayc ${ }^{c}$ Felix Kluge $^{b}$ \\ Holger Hoefling $^{a} \quad$ Martin Ullrich ${ }^{b} \quad$ Arne Küderle $^{b} \quad$ Bjoern M. Eskofier ${ }^{b}$ \\ Arne Müller ${ }^{a}$ \\ a Novartis Institutes of Biomedical Research, Novartis Pharma AG, Basel, Switzerland; \\ ${ }^{b}$ Machine Learning and Data Analytics Lab, Department of Computer Science,

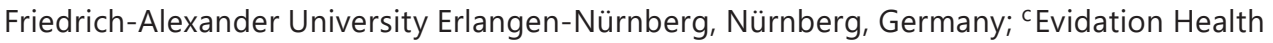 \\ Inc., San Mateo, CA, USA
}

\section{Keywords}

Step detection · Gait monitoring · Autocorrelation · Inertial sensor · Waist-worn

\section{Abstract}

Analyzing human gait with inertial sensors provides valuable insights into a wide range of health impairments, including many musculoskeletal and neurological diseases. A representative and reliable assessment of gait requires continuous monitoring over long periods and ideally takes place in the subjects' habitual environment (real-world). An inconsistent sensor wearing position can affect gait characterization and influence clinical study results, thus clinical study protocols are typically highly proscriptive, instructing all participants to wear the sensor in a uniform manner. This restrictive approach improves data quality but reduces overall adherence. In this work, we analyze the impact of altering the sensor wearing position around the waist on sensor signal and step detection. We demonstrate that an asymmetrically worn sensor leads to additional odd-harmonic frequency components in the frequency spectrum. We propose a robust solution for step detection based on autocorrelation to overcome sensor position variation (sensitivity $=0.99$, precision $=0.99$ ). The proposed solution reduces the impact of inconsistent sensor positioning on gait characterization in clinical studies, thus providing more flexibility to protocol implementation and more freedom to participants to wear the sensor in the position most comfortable to them. This work is a first step towards truly position-agnostic gait assessment in clinical settings. 


\section{Introduction}

Various neurological and musculoskeletal diseases result in motor impairments that negatively affect human locomotion [1]. Changes in human gait can be monitored through a variety of parameters including walking speed [2,3], step length [4, 5], or cadence [6]. Besides the analysis of human gait in special gait laboratories, inertial measurement units can be used to monitor gait in free-living, real-world environments [7]. Small body-mounted sensors represent a promising alternative to specialized gait analysis facilities, enabling unobtrusive long-term monitoring in the subject's habitual environment. Therefore, these sensors are increasingly used in clinical studies [8, 9], even though the analysis of data derived from such wearable sensors remains challenging [10]. A critical human factor for the reliability of sensor-based gait analysis is the sensor wearing position, as it strongly impacts the recorded signal and the level of comfort during wearing. A sensor position close to the body's center of mass, for example a belt buckle [11], allows for an easier transformation and interpretation of the inertial measurement unit signal when compared to other positions, such as the wrist, which has more dynamic and unpredictable movements [12]. The wrist is generally more comfortable than the ankle or foot, which has been shown to be most accurate for measuring gait parameters [13]. A good compromise of signal periodicity, stability, and wearing comfort might be a sensor position around the waist [11].

In a real-world environment, the correct sensor attachment is dependent upon the subjects' commitment, discipline, and functional ability to comply with the provided instructions, which is in turn dependent on the burden such as mounting complexity and the level of comfort to adhere to wearing instructions over a long period and possibly on the type of clothing (e.g., when worn on a belt around the waist). When a subject wears a sensor over multiple weeks, it is likely that they unintentionally change the sensor wearing position from day to day or deliberately change the position to improve the comfort of wearing or for other reasons. Medically relevant changes in a subject's gait over time could therefore be confounded by systematic as well as short-term frequent changes in wearing position.

A non-central sensor attachment around the waist leads to a different sensor displacement during left and right steps and introduces additional frequencies to the spectrum, in particular odd harmonic frequencies that are also observed during asymmetric gait [14]. How severely the sensor position affects gait analysis is influenced by the underlying algorithm and in particular impacts frequency-based methods [15] that describe gait as a quasiperiodic signal in which successive steps generate a repeating pattern over time [16]. The frequency spectrum changes when the sensor wearing position is altered [17]. In the patient's real world, it would be desirable to allow flexibility in sensor wearing positions to increase the subject's compliance and wearing comfort while achieving reliable analysis of the data to avoid confounding of clinical and technical changes.

In this study, we systematically investigated how changing sensor position around the waist affects the recorded signal, and how these changes impact frequency-based step detection which is fundamental for continuous gait monitoring. Based on harmonic frequency analysis we explain the differences in sensor position and explore an autocorrelation algorithm for the reliable estimation of steps for different sensor positions. This work is a first step in sensor position agnostic assessment of gait characteristics, enabling greater flexibility in protocol implementation and collection of more robust results from performance and behavioral gait assessments.

\section{Karger'}


Fig. 1. Sensor wearing positions around the waist. SREF, center of mass, on belt buckle; S0, in close proximity to SREF (distance of 3-5 cm); S45, anterior superior iliac spine; S90, outside hip; S180, sacrum (center of mass, back).

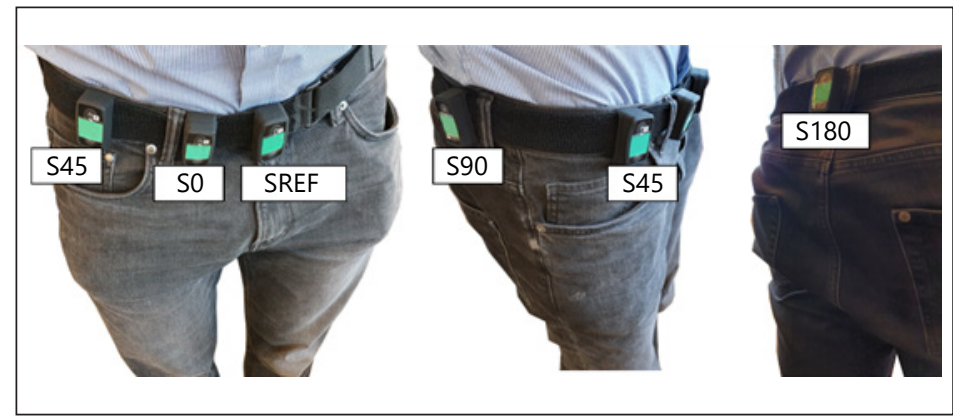

Table 1. Study demographics

\begin{tabular}{|c|c|c|c|c|c|c|c|c|}
\hline \multirow[t]{2}{*}{ Gender } & \multirow[t]{2}{*}{$n$} & \multirow{2}{*}{$\begin{array}{l}\text { Height, } \\
\mathrm{cm}\end{array}$} & \multicolumn{3}{|c|}{ Age range, $n$} & \multicolumn{3}{|c|}{ Walks, $n$ /average walking speed, $\mathrm{m} / \mathrm{s}$} \\
\hline & & & $\begin{array}{l}26-35 \\
\text { years }\end{array}$ & $\begin{array}{l}36-45 \\
\text { years }\end{array}$ & $\begin{array}{l}46-55 \\
\text { years }\end{array}$ & self-selected & turn-walk & slow \\
\hline Female & 15 & $170 \pm 6$ & 5 & 6 & 4 & $38 / 1.25$ & $38 / 1.16$ & $38 / 0.69$ \\
\hline Male & 5 & $183 \pm 4$ & 1 & 3 & 1 & $11 / 1.32$ & $11 / 1.22$ & $11 / 0.72$ \\
\hline
\end{tabular}

\section{Materials and Methods}

\section{Data Acquisition}

Twenty healthy volunteers walked a 20 -m distance in 3 different walking styles, each with up to 3 repetitions (see Table 1 for study demographics). To analyze the algorithm performance during different walking styles we included straight and turn walks at a selfselected speed and straight walks at low speed. The study had no specific inclusion criteria other than "fit for work" and the first 20 participants were selected.

A marked start and finish line predetermined the path length. The subject started and stopped walking $2 \mathrm{~m}$ behind the line to reduce the impact of acceleration and deceleration. The duration of the walk was measured with a stopwatch to ensure a walking speed difference between self-selected and slow walk styles. The tasks were performed indoors on a hard surface and with no specific shoe wear. Each subject was equipped with 11 time-synchronized inertial sensors (Physilog 5, sampling frequency $128 \mathrm{~Hz}$, GaitUp SA, Lausanne, Switzerland): $1 \times$ right shoe, $1 \times$ left shoe, $5 \times$ hip, $1 \times$ right pocket, $1 \times$ collar, $1 \times$ upper arm, $1 \times$ wrist. The sensors were attached by the subject with instructions from the study tester. The SREF sensor was placed with particular care to ensure its correct positioning, while for the other sensors we allowed slight individual changes as expected in a real-world scenario. For this work, we restrict our analysis to sensors worn around the waist (Fig. 1), but data from all positions are available for download [18].

SREF represents the sensor position for which the step detection algorithm was originally designed [19] and to which we compare the tri-axial acceleration data from the other sensors. One subject had to be excluded due to a wrong sensor attachment. In total, 147 unique walks were recorded. No participant stated any health-related issues or gait impairments. Detailed data processing is described in the online supplementary materials (for all online suppl. material, see www.karger.com/doi/10.1159/000511611). 


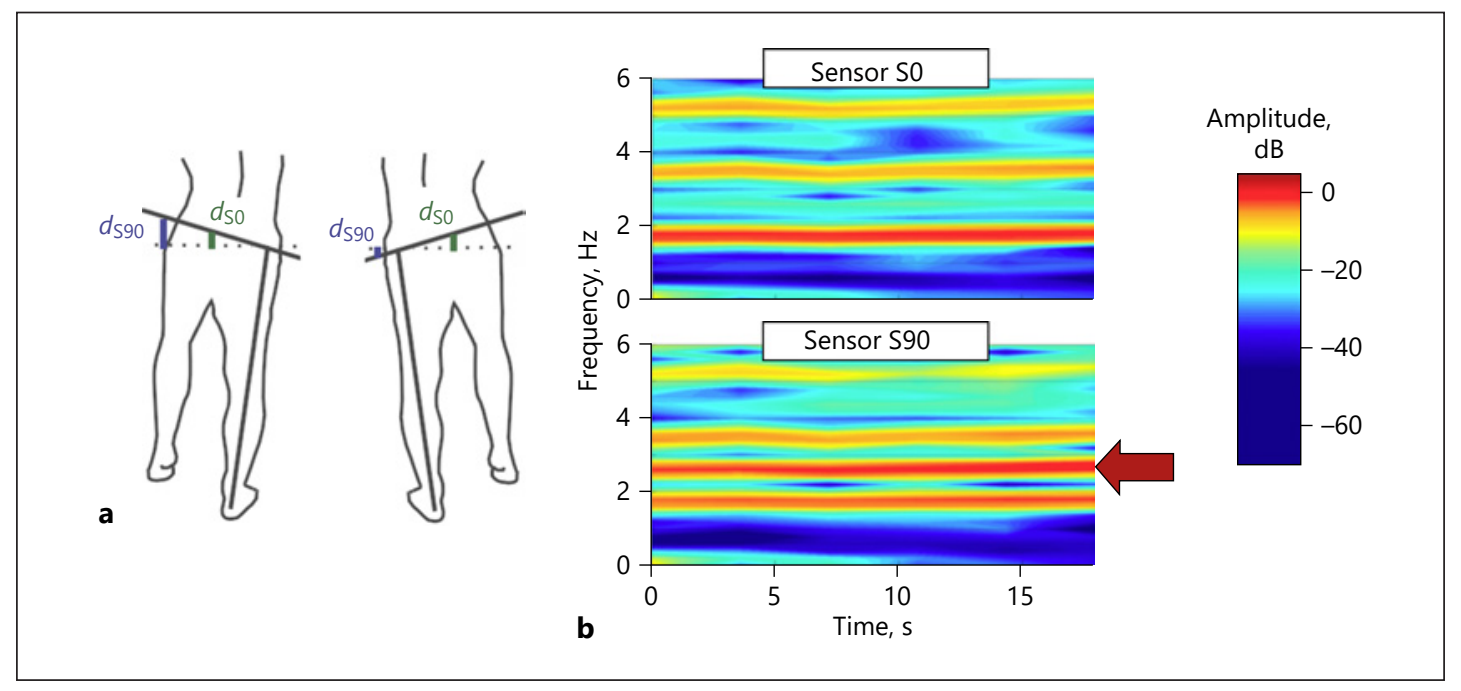

Fig. 2. a Model illustrating sensor displacement during walking (vertical axis). The sensor displacement during a left and a right step is equal for a central sensor position $\left(\mathrm{d}_{\mathrm{S} 0}\right)$. A sensor attached to the outside experiences a different sensor displacement during a left and a right step $\left(\mathrm{d}_{\mathrm{S} 90}\right)$. b Exemplified frequency spectrum (vertical axis) for walking with a sensor $d_{S 0}$ (top) and $d_{S 90}$ (bottom). The additional third odd harmonic frequency is indicated by the red arrow.

\section{Biomechanics and Harmonic Frequencies during Gait}

A non-central sensor wearing position alters the acceleration signal $[20,21]$ and impacts the harmonic frequency properties of the corresponding spectrum. A sensor attached close to the center of mass (SREF) experiences approximately the same displacement $\left(d_{s}\right)$ during a right or a left step in symmetric gait. The non-central sensor attachment around the waist (e.g., S90) leads to a different sensor displacement during left and right steps (Fig. 2a), introducing additional odd harmonic frequencies of the underlying stride frequency (Fig. 2b). Odd harmonic frequencies alternatingly increase and decrease the signal magnitude of right and left steps (see online suppl. materials).

\section{Frequency Analysis Based on Harmonic Theory}

The gait signal of each walk was initially filtered and a fast Fourier transformation (FFT) was applied on every axis to decompose the signal into its frequency content. The first 4 harmonic frequency components of the underlying stride frequency were extracted for every axis. The influence of the sensor position on the harmonic frequency components of all axes was analyzed with a principle component analysis.

The harmonic ratio (HR) is a measure of signal symmetry [14]. Here, HR is calculated as the sum of the even harmonics divided by the sum of odd harmonic frequency components per walk and axis.

\section{Algorithms for Gait Analysis}

Starting from our previously published FFT-based gait algorithm [19] we developed an autocorrelation-based step estimation with the aim of overcoming problems with frequencies introduced by asymmetric sensor wearing positions and compared performance between the 2 algorithms. The FFT-based approach utilizes the dominant frequency of the 3D signal for step detection. The autocorrelation approach works similarly but uses the fundamental (lowest) frequency of all 3 axes. Detailed descriptions of these algorithms can be found in the online supplementary materials.

\section{Karger'}


Fig. 3. Principal component analysis of the first 4 harmonic frequencies per axis. The first 2 components explain $52 \%$ of the variance. Each data point represents a single walk (color coded by sensor position). The ellipses mark the $68 \%$ CI. The arrows indicate the loading vectors (harmonic frequency components $f 2-5$ for the vertical, medio-lateral, and anterior-posterior axes $x, y$, and $z$ ).

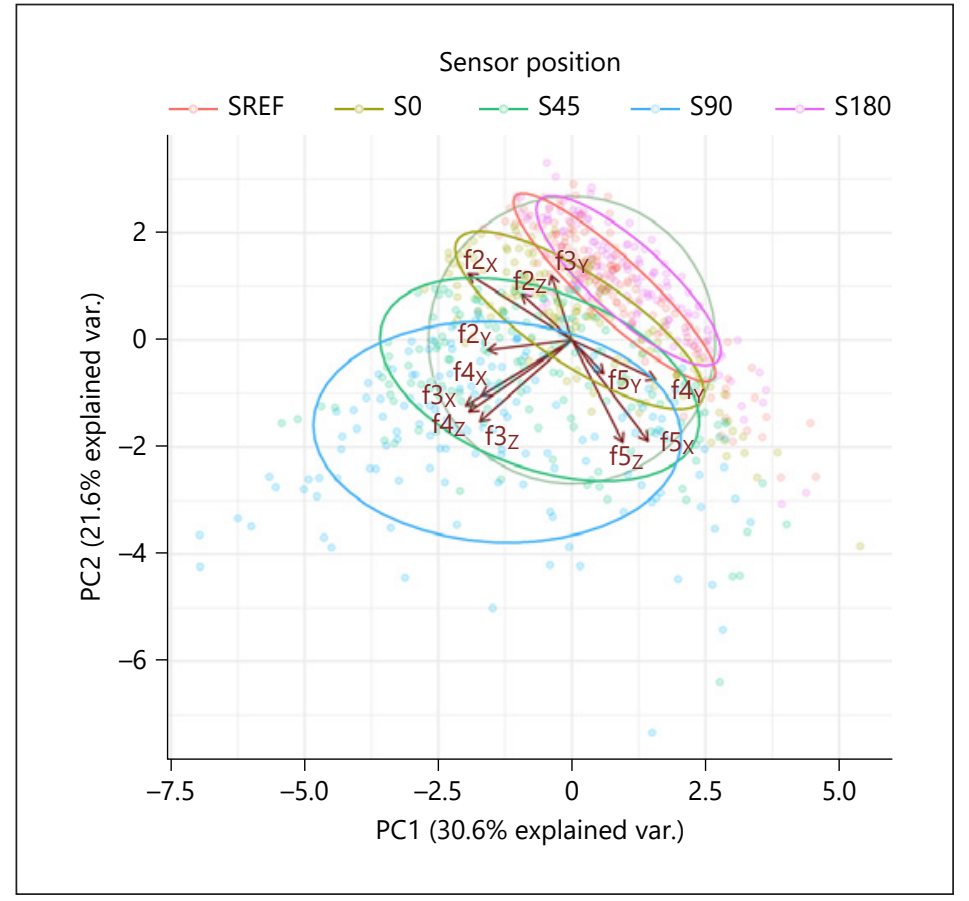

Algorithm Performance Assessment

The step detection was evaluated based on the already validated SREF sensor [19]. For this purpose, the time stamps of the detected steps were compared between SREF and the other sensors per walk with a maximum delay of $0.3 \mathrm{~s}$ accounting for synchronization imprecision and overall resulting in best performance. An SREF step was matched by identifying the corresponding step with the lowest temporal difference for the other positions. True positive (TP) steps are those matching between the 2 sensors, false positives (FP) are steps in the sensor not matched by SREF steps, and false negatives (FN) are steps in SREF that are not matched by the other sensors. We used a critical success index (CSI; calculated as TP divided by the sum of TP, FP, and FN) as the performance measure.

\section{Results}

Impact of Wear Position on Harmonic Frequency Components

Principal component analysis was applied on the first 4 harmonic frequency components per axis and for each walk (shown in Fig. 3). Sensors attached centrally (SREF, S0, S180) show strong overlap, while the centroids of sensors attached on the outside of the hip (S45 and S90) are in a separate group and show higher spread than the central positions. The third harmonic frequency (odd harmonic) of the vertical and the anterior-posterior axes have the highest magnitude and orthogonal direction and explain most of the variance between the signals of the sensor positions. The high spread in S45 and S90 is likely due to larger differences in sensor placement between participants and subject-specific gait characteristics impacting the outer positions more than the central positions.

Figure 4 shows the distribution of the inverse HR for all subjects and sensors for the vertical axis. The HR and thus the asymmetry increases with attachment distance from the center of mass (note that the position on the back at $180^{\circ}$ is also near the center of mass). 
Digital

Biomarkers
Digit Biomark 2020;4(suppl 1):50-58

Fig. 4. Inverse HR grouped by subject and colored by sensor position for the vertical axis. Each data point is for a single walk and sensor grouped by subject.

\section{\begin{tabular}{l|l}
\hline DOI: $10.1159 / 000511611$ & ( 2020 The Author(s). Published by S. Karger AG, Basel
\end{tabular} www.karger.com/dib}

Tietsch et al.: Robust Step Detection from Different Waist-Worn Sensor Positions

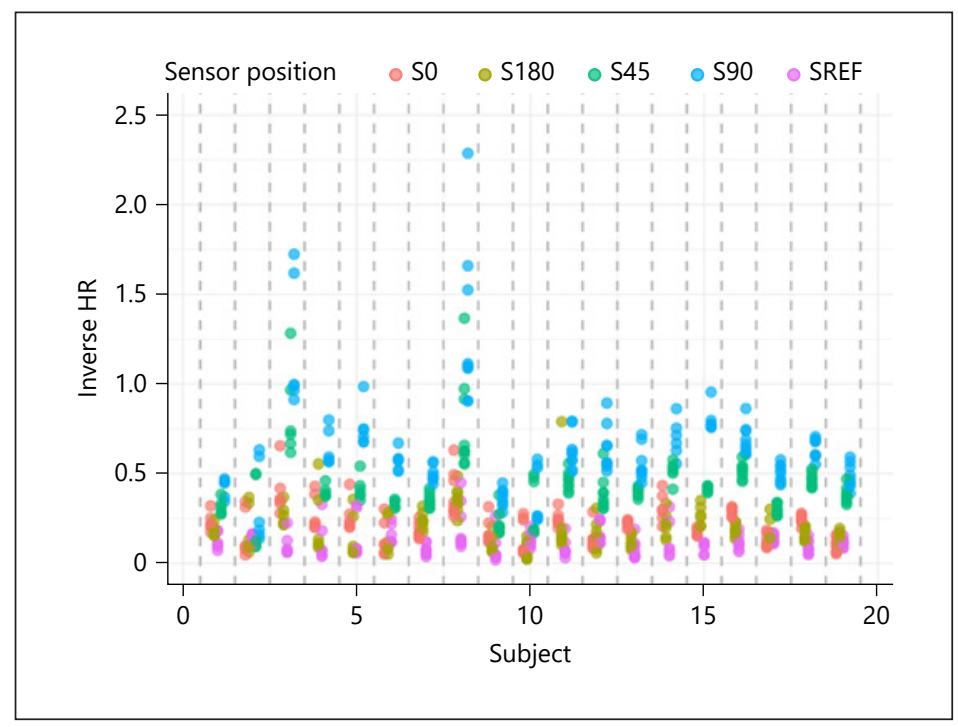

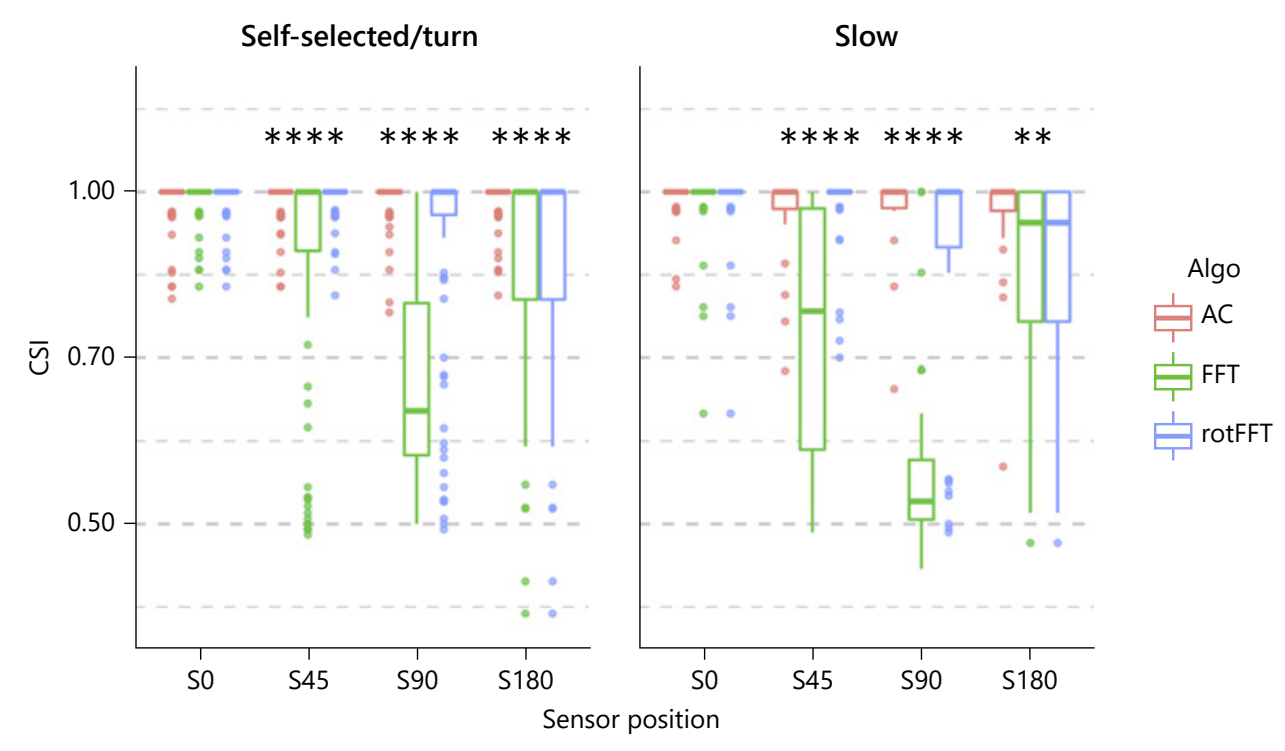

Fig. 5. Step-detection performance by sensor position, walk type (self-selected and turn walk are pooled) and algorithm given by critical performance index (CSI = TP/[TP + FP + FN $]$; see Materials and Methods for details). Significance was calculated by a Kruskal-Wallis test per sensor position and between algorithms ${ }^{* * * *} p \leq 0.0001,{ }^{* * *} p \leq 0.001,{ }^{* *} p \leq 0.01$, and no asterisk indicates $p>0.05$ ). AC, autocorrelation; FFT, unrotated data analyzed with FFT; rotFFT, data rotated according to prior knowledge of the sensor position before FFT-based step detection.

However, the variability of the HR and the overlap in positions is to some extend subject dependent (e.g., subjects 3 and 8). The HR of the anterior-posterior axis shows a similar pattern whereas the medio-lateral axis has no clear pattern and shows a higher variance (see online suppl. figures). 


\section{Assessment of Step Detection Accuracy}

Figure 5 shows the step detection performance per sensor and walk type for the FFTbased and our autocorrelation-based algorithm. As the sensor is positioned further away from the belt buckle position, the step detection accuracy of the FFT algorithm significantly decreases (green boxes). The FFT assumes a specific axis rotation, in our case a center of mass positioning, that can only be estimated with limited certainty in a real-world study. To demonstrate the impact of axis rotation, we included results obtained from the FFT method on "rotated" data, which has been preprocessed to reorientate the signal to the expected "belt buckle" center of mass orientation. Rotating the data substantially improves the performance of the FFT algorithm at all positions except S180 (blue boxes), but precise sensor data rotation is only possible if the wearing position is a priori known. Therefore, in a real-world setting the determination of the precise rotation is challenging. The autocorrelation method performs highly accurately for all positions without requiring a priori data rotation (red boxes). The performance of both methods is slightly lower during slow walks. The performance of straight walks and turn walks at self-selected speed were almost identical and are therefore pooled in Figure 5 (also see online suppl. Table 1). Overall, the autocorrelation achieves a CSI of 0.99 (across walks) compared to 0.84 for the position-specific FFT and 0.96 for the a priori rotated FFT. The average sensitivity and precision across walks and asymmetric sensor positions were both $>0.99$ for the autocorrelation and almost 0.99 for the rotated FFT, compared to 0.92 and 0.9 , respectively, for the FFT.

\section{Discussion}

Our study shows that altering the sensor wearing position systematically affects step detection of frequency-based algorithms. We propose an autocorrelation-based approach, which eliminates the need for sensor data rotation, to determine the underlying gait frequency more reliably. This approach robustly detects steps for different sensor positions around the waist and allows more flexibility in clinical study protocols to monitor gait continuously with increased analyzable wear time in real-world studies.

The analysis of the harmonic gait frequencies has revealed clear differences between the various sensor positions and shows that the dominant signal frequency does not always represent the gait frequency for asymmetric sensor positions. With increasing distance from the center of mass the magnitude of odd harmonic frequencies increases too, explaining most of the asymmetry in the signal. However, the source of asymmetry can originate from a noncentral wearing position as well as from gait asymmetry, for example due to pathological gait, such as limping. Therefore, gait asymmetry is confounded with asymmetry in sensor location, and we recommend a central attachment when analyzing gait asymmetries. Note, none of the study participants had any known gait impairment, and this is not representative of an elderly population, for example. In this work we could not analyze asymmetric gait together with sensor displacement [22]. Also, algorithm parameters were optimized based on the walks of a few subjects, and we cannot exclude possible overfitting effects. An additional validation study, possibly including pathological gait, is needed to investigate the generalizability.

We have not yet characterized the data from the additional wearing positions from our study (feet, wrist, pocket, upper trunk). These sensor positions also generate a characteristic harmonic frequency pattern during human gait [23, 24]. It is unclear whether the autocorrelation can robustly delineate steps from these sensor positions.

Our results are based on a study conducted under controlled conditions and were performed in a laboratory setting. Measuring gait in the real world poses several problems, such as unknown context and distinguishing gait periods from other activities to avoid FPs

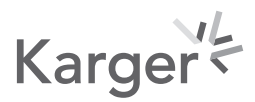


$[10,25]$. In a long-term, real-world setting, sensor position can change and may impact data analysis and study conclusions. It is therefore necessary to develop robust analytical methods that cope with flexible low-burden study protocols. The Mobilise-D consortium [28] aims to develop robust digital mobility endpoints collected in the patient's real world over the upcoming years.

\section{Acknowledgements}

We thank our colleagues Sarah Hemsley, Ruth Frommherz, and Roland Huber for their help in setting up the study, and Jason Laramie, Anna Kostikova, and Scott Kennedy for their support. We would also like to thank all study participants.

\section{Statement of Ethics}

Data were collected as part of a single site, non-interventional study carried out at the Novartis Institutes for Biomedical Research (NIBR) with Novartis employees. The Ethics Committee involved in the study granted approval and written consent was given by every participant.

\section{Conflict of Interest Statement}

Ar.M., A.K., F.K., M.U., and B.M.E. are members of the IMI Mobilise-D consortium. I.C. is an employee of and holds stock options in Evidation Health. He has received payment for lecturing on Digital Health at the ETH Zurich and FHNW Muttenz. He is an Editorial Board Member at Karger Digital Biomarkers and a founding member of the Digital Medicine Society. H.H., Am.M., and Ar.M are employees of Novartis and hold stock in Novartis. M.T. is a Novartis employee and a student at University Erlangen-Nürnberg. B.M.E. reports grants outside the submitted work from Adidas AG, Agaplesion gAG, and Bosch Sensortec GmbH. He received compensation from lecturing for AbbVie Deutschland GmbH \& Co. KG and Agaplesion gAG. He is a co-founder and co-owner of Portabiles GmbH and Portabiles HealthCare Technologies $\mathrm{GmbH}$, and co-inventor of gait analysis patent application EP 16174268.9. F.K., M.U., and A.K. received funding from IMI Mobilise-D (grant agreement 820820).

\section{Funding Sources}

This work was sponsored by Novartis Pharma AG and received no direct funding.

\section{Author Contributions}

M.T., Am.M, H.H., F.K., and Ar.M. contributed to the design of data collection and performed analysis and interpretation. I.C. ran the data acquisition. M.U., F.K., and A.K. contributed ideas to the autocorrelation. Ar.M., M.T., and I.C. drafted the manuscript. H.H., F.K., A.K., M.U., Am.M., and B.M.E. revised the manuscript.

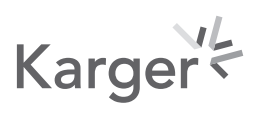




\section{References}

1 Morris R, Lord S, Bunce J, Burn D, Rochester L. Gait and cognition: mapping the global and discrete relationships in ageing and neurodegenerative disease. Neurosci Biobehav Rev. 2016 May;64:326-45.

2 Fritz S, Lusardi M. White paper: "walking speed: the sixth vital sign”. J Geriatr Phys Ther. 2009;32(2):46-9.

3 Studenski S, Perera S, Patel K, Rosano C, Faulkner K, Inzitari M, et al. Gait speed and survival in older adults. JAMA. 2011 Jan;305(1):50-8.

4 Mak MK. Reduced step length, not step length variability is central to gait hypokinesia in people with Parkinson's disease. Clin Neurol Neurosurg. 2013 May;115(5):587-90.

5 Mbourou GA, Lajoie Y, Teasdale N. Step length variability at gait initiation in elderly fallers and non-fallers, and young adults. Gerontology. 2003 Jan-Feb;49(1):21-6.

6 Tudor-Locke C, Schuna JM Jr, Han H, Aguiar EJ, Larrivee S, Hsia DS, et al. Cadence (steps/min) and intensity during ambulation in 6-20 year olds: the CADENCE-kids study. Int J Behav Nutr Phys Act. 2018 Feb;15(1):20.

7 Ferrete Ribeiro N, Santos CP. Inertial measurement units: a brief state of the art on gait analysis. IEEE 5th Portuguese Meeting on Bioengineering; 2017; Coimbra. p. 1-4.

8 Yang S, Li Q. Inertial sensor-based methods in walking speed estimation: a systematic review. Sensors. 2012; 12(5):6102-16.

9 Mueller A, Hoefling HA, Muaremi A, Praestgaard J, Walsh LC, Bunte O, et al. Continuous Digital Monitoring of Walking Speed in Frail Elderly Patients: Noninterventional Validation Study and Longitudinal Clinical Trial. JMIR Mhealth Uhealth. 2019 Nov;7(11):e15191.

10 Khandelwal S, Wickström N. Gait Event Detection in Real-World Environment for Long-Term Applications: Incorporating Domain Knowledge Into Time-Frequency Analysis. IEEE Trans Neural Syst Rehabil Eng. 2016 Dec;24(12):1363-72.

11 Schimpl M, Lederer C, Daumer M. Development and validation of a new method to measure walking speed in free-living environments using the actibelt ${ }^{\circledR}$ platform. PLoS One. 2011;6(8):e23080.

12 Meyns P, Bruijn SM, Duysens J. The how and why of arm swing during human walking. Gait Posture. 2013 Sep; 38(4):555-62.

13 Simpson LA, Eng JJ, Klassen TD, Lim SB, Louie DR, Parappilly B, et al. Capturing step counts at slow walking speeds in older adults: comparison of ankle and waist placement of measuring device. J Rehabil Med. 2015 Oct; 47(9):830-5.

14 Bellanca JL, Lowry KA, Vanswearingen JM, Brach JS, Redfern MS. Harmonic ratios: a quantification of step to step symmetry. J Biomech. 2013 Feb;46(4):828-31.

15 Chidean MI, Barquero-Pérez Ó, Goya-Esteban R, Sánchez Sixto A, de la Cruz Torres B, Naranjo Orellana J, et al. Full Band Spectra Analysis of Gait Acceleration Signals for Peripheral Arterial Disease Patients. Front Physiol. 2018 Aug; 9:1061.

16 Sabatini AM, Ligorio G, Mannini A. Fourier-based integration of quasi-periodic gait accelerations for drift-free displacement estimation using inertial sensors. Biomed Eng Online. 2015 Nov;14(1):106.

17 MacDougall HG, Moore ST. Marching to the beat of the same drummer: the spontaneous tempo of human locomotion. J Appl Physiol. 2005 Sep;99(3):1164-73.

18 Tietsch M, Mueller A. Robust step detection from different waist-worn sensor positions - implications for clinical studies. Zenodo. 2020. https://doi.org/10.5281/zenodo.3952045.

19 Keppler AM, Nuritidinow T, Mueller A, Hoefling H, Schieker M, Clay I, et al. Validity of accelerometry in step detection and gait speed measurement in orthogeriatric patients. PLoS One. 2019 Aug;14(8):e0221732.

20 Sayeed T. Methods and models in signal processing for gait analysis using waist-worn accelerometer: a contribution to Parkinson's disease [PhD thesis]. Barcelona: University of Catalonia; 2015.

21 Wark T, Karunanithi M, Chan W. A Framework for Linking Gait Characteristics of Patients with Accelerations of the Waist. In Annual International Conference of the IEEE Engineering in Medicine and Biology Society; 2005.

22 Lowry KA, Lokenvitz N, Smiley-Oyen AL. Age- and speed-related differences in harmonic ratios during walking. Gait Posture. 2012 Feb;35(2):272-6.

23 Ullrich M, Küderle A, Hannink J, Din SD, Gasner H, Marxreiter F, et al. Detection of Gait From Continuous Inertial Sensor Data Using Harmonic Frequencies. IEEE J Biomed Health Inform. 2020 Jul;24(7):1869-78.

24 Fasel B, Duc C, Dadashi F, Bardyn F, Savary M, Farine PA, et al. A wrist sensor and algorithm to determine instantaneous walking cadence and speed in daily life walking. Med Biol Eng Comput. 2017 Oct;55(10):177385.

25 Hickey A, Del Din S, Rochester L, Godfrey A. Detecting free-living steps and walking bouts: validating an algorithm for macro gait analysis. Physiol Meas. 2017 Jan;38(1):N1-15.

26 Hausamann P, Daumer M, MacNeilage PR, Glasauer S. Ecological momentary assessment of head motion: toward normative data of head stabilization. Front Hum Neurosci. 2019 Jun;13:179.

27 Moe-Nilssen R, Helbostad JL. Estimation of gait cycle characteristics by trunk accelerometry. J Biomech. 2004 Jan;37(1):121-6.

28 Rochester L, Mazzà C, Mueller A, Caulfield B, McCarthy M, Becker C, et al. A roadmap to inform development, validation and approval of digital mobility outcomes: the Mobilise-D approach. Digit Biomark. Forthcoming 2020. DOI: $10.1159 / 000512513$ 